\title{
Islamistes et rebelles touaregs maliens : alliances, rivalités et ruptures
}

Emmanuel Grégoire

\section{(2) OpenEdition}

Journals

Édition électronique

URL : http://journals.openedition.org/echogeo/13466

DOI : 10.4000/echogeo.13466

ISSN : 1963-1197

Éditeur

Pôle de recherche pour l'organisation et la diffusion de l'information géographique (CNRS UMR 8586)

\section{Référence électronique}

Emmanuel Grégoire, «Islamistes et rebelles touaregs maliens : alliances, rivalités et ruptures », EchoGéo [En ligne], Sur le Vif, mis en ligne le 03 juillet 2013, consulté le 19 avril 2019. URL : http:// journals.openedition.org/echogeo/13466; DOI : 10.4000/echogeo.13466

Ce document a été généré automatiquement le 19 avril 2019

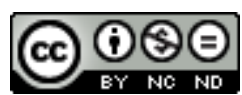

EchoGéo est mis à disposition selon les termes de la licence Creative Commons Attribution - Pas d'Utilisation Commerciale - Pas de Modification 4.0 International 


\title{
Islamistes et rebelles touaregs maliens : alliances, rivalités et ruptures
}

\author{
Emmanuel Grégoire
}

1 La décision (10 janvier 2013) aussi brutale qu'inattendue du président François Hollande d'intervenir au Mali avec l'aval de l'ONU (décision n²085) marque le retour de la France sur la scène africaine. Celui-ci a été d'autant plus réussi que l'opération Serval fut approuvée par les chefs d'État africains et vite marquée par une série de succès militaires : à une guerre éclair menée par des rebelles touaregs et des groupes islamistes qui s'emparèrent en trois jours de Kidal, Gao et Tombouctou (30, 31 mars et $1^{\text {er }}$ avril 2012), la France répliqua par une offensive toute aussi foudroyante qui les en chassa (janvierfévrier 2013). Elle rétablit ensuite la souveraineté de l'État malien sur l'ensemble du territoire national à l'exception de la région de Kidal. L'accord de paix signé le 18 juin 2013 entre des représentants des autorités de Bamako et de la rébellion touarègue devrait permettre le prochain retour de l'armée malienne dans la ville et l'organisation de l'élection présidentielle fixée au 28 juillet.

2 Au moment où le conflit semble trouver une solution (fin juin 2013), il convient de prendre un peu de recul en revenant sur les alliances, les rivalités et les ruptures entre et au sein des différents mouvements à l'origine de la crise. Inhérentes à leurs dynamiques internes, ces divisions expliquent en partie leur récent échec politique, militaire et religieux. Nous analyserons d'abord les groupes « terroristes » emmenés par des salafistes et jihadistes algériens qui, chassés de leur pays au terme de la guerre civile (1991-2002), se réfugièrent au Nord Mali (2003), ce qui le déstabilisa. Nous nous pencherons ensuite sur les rebelles touaregs une nouvelle fois désunis. Depuis les précédentes révoltes, leurs mouvements se sont cependant radicalisés, puisque des Touaregs du MNLA (Mouvement national de libération de l'Azawad) proclamèrent l'indépendance de l'Azawad (6 avril 2012) tandis que les jihadistes d'Ansar ed-Dine (« Partisans de la foi ») voulaient créer par la lutte armée un État islamique touareg appliquant la sharia. Nous nous demanderons 
enfin pourquoi les Touaregs nigériens n'ont pas pris les armes alors que les rébellions malienne et nigérienne ont toujours présenté de grandes similitudes.

Avant d'entrer dans le vif du sujet, une remarque d'ordre méthodologique: l'analyse repose sur le dépouillement de la presse malienne, nigérienne et française, la consultation de sites internet, quelques entretiens menés à Paris auprès de personnes bien informées ainsi que sur ma connaissance de l'espace sahélo-saharien. L'insécurité qui y prévaut interdit en effet tout travail de terrain et me contraint à une démarche différente de mon approche habituelle (anthropologique et géographique).

\section{La mouvance islamiste au Mali : implantation, rivalités et divisions}

4 Des dissensions au sein du GIA (Groupe islamique armé) qui commit les pires massacres de la guerre civile algérienne conduisirent à son éclatement (1998) et à la création du GSPC (Groupe salafiste pour la prédication et le combat) par Abdel Malek Droukdal qui entendait poursuivre le jihad contre les militaires afin d'établir un État islamique. En 2006, le GSPC fit allégeance à Al-Qaïda d'Oussama Ben Laden et devînt, en 2007, AQMI (AlQaïda au Maghreb islamique) avec pour émir Abdel Malek Droukdal, véritable penseur du mouvement.

5 Pour compenser ses revers militaires dans le nord de l'Algérie, une frange d'AQMI se replia sur le flanc sud du pays et au Sahel malien. Son objectif n'était pas de conquérir le pouvoir d'État des pays subsahariens, mais d'instaurer une zone de non droit pour se livrer en toute impunité à toutes sortes de trafics, d'appliquer la sharia dans toute la bande sahélo-saharienne et d'étendre son influence en constituant un émirat doté d'un immense territoire qui couvrirait une large partie de l'extrême sud-ouest algérien, du nord de la Mauritanie, du Mali et du Niger jusqu'aux confins du lac Tchad. Dans cette configuration, trois grandes katibas (bataillons) furent mises en place (Bourgeot, 2011) : à l'Ouest, celle de Mokhtar Ben Belmokhtar, trafiquant connu de cigarettes et de drogue ; à l'Est (massif des Ifoghas), celle d'Abou Zeid, contrebandier et preneur d'otages notoire ; au Nord, dans l'oasis de Taoudéni, celle de Yahia Djouadi (alias Abou Hammar), qui devint le bras droit d'Abdel Malek Droukdal puis fut nommé émir du Sahara en septembre 2012.

6 L'implantation de ces salafistes algériens dans le septentrion malien il y a dix ans bénéficia de la passivité du président Amadou Toumani Touré, alors qu'ils auraient probablement pu être aisément repoussés car peu nombreux (300 à 500 hommes). Ce laissez-faire leur a permis d'y faire parfois souche en épousant des femmes touarègues ou arabes maliennes, comme c'est le cas de Mokhtar Ben Belmokhtar, de grossir leurs rangs en recrutant des Subsahariens et d'accroître leur emprise territoriale. Parallèlement, ils renforcèrent leur armement grâce à l'argent gagné sur le paiement de rançons en contrepartie de la libération d'otages occidentaux, sur divers trafics et à partir de la distribution massive d'armes à laquelle le colonel Kadhafi se livra, en 2011, pour lutter contre les insurgés de Benghazi et les forces de l'OTAN. AQMI se dota alors d'armes lourdes et de moyens de transmission sophistiqués tandis que les Touaregs maliens et nigériens, dont plusieurs centaines combattirent aux côtés des partisans du guide de la révolution, s'équipèrent en armes légères avec l'objectif de s'en servir de retour au pays (Grégoire \& Bourgeot, 2011). 
7 Du point de vue politique, des divergences traversent la mouvance islamiste, marquée par des rivalités entre émirs. Fin 2011, ces dissensions donnèrent naissance au Mouvement pour l'unité et le jihad en Afrique de l'Ouest (MUJAO), à la suite de l'enlèvement en Algérie de trois Européens (octobre 2011) à l'initiative du Mauritanien Hamada Ould Mohamed Kheira. Lorsque celui-ci et son second, Abou Ali, un Algérien d'origine malienne, présentèrent les trois otages aux émirs installés dans les montagnes de Tigharghar (massif des Ifoghas) une violente dispute éclata : inquiets que des éléments d'AQMI soient intervenus en Algérie, Abou Zeid et Yahia Djouadi désapprouvèrent l'opération et donnèrent l'ordre à leurs auteurs de rapatrier les otages de l'autre côté de la frontière'. Ces derniers refusèrent, accusant les deux émirs d'être de faux jihadistes, de s'enrichir sur le dos de leurs combattants à travers les rançons et les trafics et d'investir uniquement en Algérie ${ }^{2}$. Pour Ould Mohammed Kheira et Abou Ali, ceux qui ne sont pas Algériens ne profitent pas de ces "business ». C'est ainsi qu'ils partirent s'installer avec leurs partisans et leurs otages dans les régions de Gao, devenue leur fief et de Ménaka. La rupture était consumée. Rejoints par des arabes maliens, le MUJAO agissait désormais pour son propre compte (rapts d'Occidentaux, trafic d'armes et de drogue ${ }^{3}$, etc.) et procéda à de nombreux recrutements, notamment parmi les Peuls de la vallée du Niger (et parmi eux des Nigériens) sur une base à la fois islamiste et anti-touareg ou anti-MNLA, celui-ci ayant commis de nombreuses exactions dans la Vallée.

La création du MUJAO affaiblit AQMI, dont Ould Mohammed Kheira était un des hommes clés ayant participé à de nombreuses opérations au nom de l'organisation ${ }^{4}$. Le 9 décembre 2012, le MUJAO fut à son tour touché par un mouvement de dissidence avec l'apparition d'Ansar al-Sharia (défenseurs de la loi islamique) fondé à Gao par des islamistes membres de la tribu barabiche de Tombouctou liés à une milice libyenne du même nom. Au même moment, Mokhtar Ben Belmokhtar créa son propre groupe, les "Signataires par le sang ", s'émancipant ainsi d'AQMI qu'il ne se privait pas de critiquer sur des forums et de courtcircuiter, en tentant d'entrer directement en contact avec les hauts responsables d'AlQaïda en Afghanistan. Il se rapprocha alors du MUJAO et rompit ses relations avec Abou $Z_{\text {Zid }}^{5}$, avec lequel il était en désaccord et se livrait à une véritable course à la prise d'otages. Ces scissions en cascade montrent que le mouvement salafiste et jihadiste n'est ni homogène ni uni. Des clivages politiques et économiques existent entre, d'une part, Algériens, Marocains et Libyens qui contrôlent les grandes katibas et en occupent les postes importants et, d'autre part, Subsahariens et Sahraouis qui s'estiment lésés quant à la répartition des rentes monopolisée.s par les premiers ${ }^{6}$ tandis que les seconds en perçoivent les miettes : la mouvance islamiste est en effet hiérarchisée entre ceux (les Blancs) qui commandent et ceux (les Noirs) qui sacrifient leur vie au combat au nom de l'islam. Dans des pages fort intéressantes, Judith Scheele (2012) décrit les hiérarchies raciales qui prévalent à Tamanrasset : au sommet de la pyramide se trouvent les Algériens blancs puis les Touaregs, les Arabes maliens et les Maures, les Algériens noirs et enfin les Subsahariens qui en occupent la base. Ces hiérarchies qui renvoient à l'histoire précoloniale régionale (traite des esclaves) structurent aussi les groupes islamistes: le racisme a poussé de nombreux Subsahariens à déserter, ne voulant pas servir de chair à canons comme cela se serait produit lors de l'offensive d'AQMI sur l'aéroport de Sévaré (début janvier 2013), où des combattants noirs auraient été placés en première ligne en raison du risque d'intervention étrangère. De même, des Subsahariens ont été abandonnés dans l'Adrar des Ifoghas par leurs chefs algériens qui avaient pris la fuite. Ils 
furent faits prisonniers par les militaires français, les enfants soldats capturés étant remis à des ONG.

9 Une lettre trouvée peu de temps après la reconquête de Tombouctou déplorait les destructions de mausolées et de bibliothèques ainsi que les lapidations et les amputations publiques. Son auteur ${ }^{7}$ explique que ces agissements avaient été une grave erreur car ils avaient dressé les populations contre les salafistes et les jihadistes, déjà perçus comme des étrangers en raison de l'origine algérienne d'AQMI. C'est ce qui s'est effectivement passé, comme en témoignent les scènes de liesse qui eurent lieu à Gao et à Tombouctou à l'arrivée des troupes françaises et maliennes. $\mathrm{Si}$, dans un premier temps, leurs habitants ne marquèrent pas de franche opposition à l'égard des islamistes, attendant de "voir " après avoir subi les pillages et les exactions des Touaregs du MNLA, très vite les châtiments perpétrés le plus souvent contre des " Noirs ", les destructions à Tombouctou d'édifices religieux et de livres anciens, symbole de l'islam local (malékite), l'obligation faite aux femmes de porter le voile et l'interdiction de fumer, de jouer de la musique et de se livrer à des jeux (football, loterie, etc.) suscitèrent un sentiment d'hostilité qui ne pouvait s'exprimer au grand jour par peur de représailles. Aussi le déploiement massif de soldats français dans la région avec pour mission d'anéantir AQMI par les armes a-t-il été favorablement accueilli alors que certains (Loncle \& Plagnol, 2012) redoutaient la désapprobation des populations, qui auraient pu voir là un affrontement entre civilisations (chrétienne et musulmane), comme les responsables islamistes l'espéraient.

L'attitude d'AQMI à l'égard des mouvements touaregs fut différente car il ne s'agissait pas de populations sans défense, mais de groupes armés qui avaient, les premiers (MNLA), pris les armes contre l'État malien. En raison de son idéologie läque, il ne pouvait être un allié et devait par conséquent être combattu. Mieux organisés et équipés, les salafistes chassèrent le MNLA des villes du Nord-Mali dont il s'était emparé au début de l'année 2012 et assurèrent ainsi leur suprématie sur plus de la moitié du pays (juin 2012). En revanche, l'alliance avec les islamistes touaregs d'Ansar ed-Dine était essentielle car elle permettait aux émirs de renforcer leur ancrage dans le massif des Ifoghas et de disperser les pressions de la communauté internationale en cas d'intervention militaire. La création (24 janvier 2013) du MIA (Mouvement islamique de l'Azawad) par des membres d'Ansar ed-Dine déjoua ce plan: AQMI et le MUJAO affrontèrent désormais seuls les troupes françaises, maliennes, tchadiennes et nigériennes, Ansar ed-Dine ayant vu la plupart de ses hommes rallier le MIA qui s'était aussitôt déclaré prêt à collaborer avec l'armée française. Les cartes furent donc redistribuées au détriment des islamistes, privés d'un précieux allié. Ce renversement d'alliance bénéficia aux forces françaises, qui trouvèrent là un nouvel appui.

\section{La persistance du « problème " touareg malien}

11 Un bref rappel des précédentes rébellions s'impose pour mieux comprendre les revendications du présent mouvement, mais aussi ses divisions.

\section{Les rébellions touarègues de 1963-1964, 1990-1992 et 2006}

$12 \mathrm{Au}$ cours de la période coloniale, la France avait accordé un statut particulier aux chefferies touarègues à travers la mise en place d'un système de gouvernance proche de l' indirect rule britannique : en s'alliant aux imenokalen (sing. amenokal) ${ }^{8}$ notamment à celui 
des Kel Adagh, les militaires français entendaient éviter d'éventuelles révoltes et soustraiter leur contrôle sur de vastes étendues inhospitalières, peu peuplées et sans intérêt économique. Le président Modibo Keita refusa de prolonger cette organisation car elle s'opposait à la nature socialiste de son régime qui entendait, au contraire, administrer directement l'ensemble du pays et briser le pouvoir des chefferies féodales, d'où une première confrontation en 1963-1964. Celle-ci se produisit dans l'Adrar des Ifoghas et ne concerna qu'une petite partie des Touaregs de l'Adagh: ceux-ci revendiquaient le maintien du rôle prépondérant dont leur aménokal bénéficiait sous l'autorité française et mettait en avant des thèmes indépendantistes. Peu préparée, la révolte se concentra sur Kidal et le nord de son arrière-pays, les Touaregs de Ménaka et de Tombouctou restant à l'écart du mouvement (Boilley, 1999 rééd. 2012). La logique de l'État-nation justifia l'écrasement de la rébellion. La répression fit de nombreuses victimes parmi les Kel Adagh mais aussi d'autres Touaregs, condamnant beaucoup d'entre eux à l'exil en Algérie pour se soustraire aux massacres de l'armée malienne.

13 La violence de la répression ne permit pas de désamorcer la crise ni surtout d'instaurer un climat de dialogue entre les deux parties, d'autant plus que la paix ne fut pas négociée, mais imposée comme le résultat d'un rapport de force défavorable aux Kel Adagh. Une méfiance réciproque et durable s'instaura alors entre les Touaregs et les populations nomades dans leur ensemble et l'État malien: à chaque épreuve traversée par la communauté (sécheresses et famines de 1973-74 puis de 1984-85, pertes de bétail, épidémies, etc.), celui-ci était accusé d'incurie et ses agents de corruption et de détournement de l'aide. Il en fut ainsi également au Niger, où un climat de défiance prévalut entre les Touaregs et le général Seyni Kountché, qui les soupçonnait de vouloir déstabiliser le pays, d'où leur mise sous surveillance après la tentative de coup d'état fomentée, en 1976, par quelques-uns d'entre eux (Grégoire, 1999 rééd. 2010). Les sécheresses poussèrent des familles maliennes et nigériennes entières à s'exiler en Algérie et en Libye, où elles rejoignirent les Kel Adagh partis en 1964. Des ishumar ${ }^{9}$ sans perspectives d'avenir s'enrôlèrent dans la légion islamique du colonel Kadhafi au sein de laquelle ils combattirent notamment au Liban, où celui-ci avait envoyé un corps expéditionnaire pour assister les Palestiniens, puis dans la bande d'Aozou (Tchad).

S'estimant marginalisés tant du point de vue politique qu'économique et toujours marqués par la terrible répression de 1963-1964, les Touaregs de l'Adagh reprirent les armes, le 29 juin 1990, avec l'attaque de la gendarmerie de Ménaka. Cette fois-ci, ils entrainèrent avec eux d'autres groupements touaregs et des nomades d'origine araboberbères qui exprimaient, eux aussi, toutes les frustrations et les problèmes qui n'avaient toujours pas été résolus depuis l'indépendance (Boilley, 1999 rééd. 2012). Les ishumar constituaient le gros des combattants. Militairement bien formés, dotés d'armes modernes et de véhicules $4 \times 4$ et connaissant parfaitement le terrain, ils infligèrent des pertes à l'armée malienne qui n'avait plus sa suprématie passée. De plus, le général Moussa Traoré était affaibli par les multiples mouvements politiques et sociaux qui exigeaient son départ. Après six mois de guérilla au cours desquels l'armée nationale fut mise en difficulté, le général Moussa Traoré proposa aux rebelles du Mouvement populaire de l'Azawad (MPA) de négocier. Acculé, il signa, sous l'égide de l'Algérie, avec Iyad ag Ghali, responsable du MPA, les Accords de paix de Tamanrasset (6 janvier 1991). Ces accords accordaient aux Touaregs l'autonomie interne et de multiples avantages, à travers un statut particulier pour les régions du Nord Mali qui ne remettait toutefois pas en cause l'unité nationale. Ils ne furent jamais appliqués en raison de l'éclatement du 
MPA en plusieurs fronts: si les ishumar étaient unis en Libye, quelles que soit leurs fractions d'origine, leurs divisions réapparurent lors du retour au pays (Boilley, 1999 rééd. 2012). Soudés dans la lutte, la rébellion se scinda en 1991 en de multiples mouvements qui avaient des bases tribales: ces divisions traduisaient l'échec de son projet politique. Sur le terrain, la difficulté de mettre en œuvre les Accords de Tamanrasset venait de l'insécurité persistante, notamment dans la boucle du Niger, théâtre de combats. Cette instabilité se prolongea jusqu'à la signature du Pacte national (11 avril 1992). Quatre ans plus tard (27 mars 1996), tous les fronts déposèrent les armes et les brûlèrent dans un impressionnant brasier (la Flamme de la paix).

Le Pacte national stipulait que la crise devait se régler dans le cadre d'un Mali unitaire. Il prévoyait l'intégration d'anciens rebelles au sein de l'armée et de l'administration et accordait un statut particulier au Nord, avec la mise en place de la décentralisation. Comportant des lourdeurs administratives et un coût élevé alors que l'État malien était désargenté, celle-ci n'a pas produit les effets escomptés, malgré l'organisation de premières élections communales en 1999. Le Pacte national devait aussi s'accompagner de gros investissements en matière de développement, ce qui fut insuffisamment fait. Cela déplut à certains chefs touaregs, qui reprochèrent au pouvoir central son laxisme. Aussi, le 23 mars 2006, une troisième rébellion éclata, avec la création de l'Alliance démocratique pour le changement (ADC). Tout comme celle de 1963-1964, la révolte fut l'initiative des Kel Adagh, à laquelle adhérèrent les autres groupes touaregs. Comme les mouvements antérieurs, elle ne remettait pas en cause les frontières du pays. En revanche elle s'inscrivait dans une conjoncture nouvelle née de l'installation d'AQMI dans la zone, mais elle se démarqua de l'organisation, allant jusqu'à l'affronter pour tenter de l'en chasser. Cette nouvelle donne et la menace qu'elle représentait pour la stabilité du pays obligèrent les autorités maliennes à résoudre rapidement la crise en s'appuyant de nouveau sur la médiation algérienne. Celle-ci déboucha sur les Accords d'Alger (juillet 2006), qui reprenaient plusieurs dispositions du Pacte national, véritable accord qui pouvait devenir la base des relations entre les populations du Nord et le reste du pays car il rendait possible l'intégration des populations touarègues et maures au sein de la nation malienne (Boilley, 1999 rééd. 2012).

Ces accords d'Alger comme les précédents accords tardèrent à se concrétiser. Quant aux opérations de développement, elles furent insuffisantes pour relancer l'économie régionale et créer des emplois. Aussi Ibrahim ag Bahanga de la tribu des Kel Adagh fondat-il en 2008 l'Alliance des Touaregs du Nord-Mali pour le changement (ATNMC) dénonçant une nouvelle fois les carences de l'État malien. Même si les opérations militaires furent réduites, l'ATNMC reposa le problème du rapport des Touaregs à l'État central. Jusqu'au déclanchement $d u$ conflit actuel, aucune solution consensuelle n'avait été trouvée ${ }^{10}$. Certains observateurs estimaient cependant que le " pire » avait été évité, à savoir une collusion entre les rebelles touaregs et les islamistes d'AQMI : autrement dit, leur alliance aurait pu se produire dès 2008 .

Cette brève rétrospective des rébellions touarègues maliennes montre qu'il y a, au départ, des processus d'alliances autour de revendications communes et d'intérêts partagés, puis des mécanismes de fragmentation des mouvements, dont certains sont calqués sur les grands lignages qui forment la société touarègue. Celle-ci est en effet marquée par des divisions, des luttes et des ressentiments entre tribus qu'elle ne parvient pas à surmonter. Ces nombreux clivages, qui renvoient aux dynamiques internes de la société, rendent difficile la construction d'un État central indépendant d'autant plus qu'à ces clivages 
horizontaux s'ajoutent des clivages verticaux entre nobles (imajeren), vassaux (imrad) et esclaves (iklan, souvent appelés Bellas). Ces deux derniers groupes sont généralement hostiles aux revendications indépendantistes et jihadistes, dont les leaders sont tous nobles. On retrouve le même cas de figure chez les Touaregs nigériens, la rébellion de 1991-1995 affichant, au départ, un front uni, pour s'émietter ensuite en de multiples fronts aux intérêts divergents (Grégoire, 1999 rééd. 2010).

$\mathrm{Au}$ Mali comme au Niger, ces rébellions sont le fait d'une petite élite politico-militaire parfois lettrée, sans légitimité élective : ses chefs qui se sont imposés lors des révoltes des années 1990 n'ont jamais été mandatés par les populations dont ils s'autoproclament porte-parole, alors que la majorité des Touaregs ne cautionne ni n'approuve leurs revendications et la lutte armée. Ces populations, qui cohabitent pacifiquement depuis très longtemps avec les autres groupes régionaux (Songhaï, Peul, Bozo, Dogon, Maures et Arabes), sont les premières victimes de leurs agissements, étant condamnées à subir les évènements et leur cortège de conséquences (exil et misère). De plus, elles ne sont jamais consultées dans les négociations de paix qui se sont toujours déroulées, au Mali comme au Niger, entre chefs rebelles et pouvoir central, alors que les notables (chefs de villages et de tribus), les élus nationaux et locaux ainsi que divers représentants des populations auraient dû y être associés. Beaucoup de Maliens critiquent ce dialogue unilatéral avec les seuls responsables rebelles, qui ne tient pas compte des autres communautés régionales ni de la majorité des Touaregs hostiles à leur projet indépendantiste. Pour ces Maliens, les concessions qui leur ont été faites, au fil des conflits, sous la forme d'un retrait progressif de l'État du Nord Mali, sont à l'origine du cycle de rébellions et de surenchères auquel on assiste, et finalement de la crise actuelle.

\section{La rébellion de 2012-2013}

Si cette nouvelle révolte a pris une grande ampleur, c'est parce que le contexte régional a profondément changé depuis 2007, avec la présence plus forte d'AQMI, la chute du colonel Kadhafi et l'essor du trafic de drogue, qui permit aux Touaregs de disposer de moyens financiers importants: beaucoup de chefs traditionnels et de rebelles en ont profité en percevant des droits de passage ou en assurant le convoyage de la précieuse marchandise.

20 À l'origine du conflit se trouve le MNLA, né à Kidal (15 octobre 2011) de la fusion du Mouvement national de l'Azawad (MNA), composé de jeunes Touaregs urbains et scolarisés et du Mouvement touareg du Nord-Mali (MTNM), une fraction armée dirigée par Ibrahim ag Bahanga. Le MNLA estimait que le pouvoir central n'avait pas respecté les engagements pris lors des Accords d'Alger, notamment concernant la décentralisation et le développement des régions nord, lesquelles connaissaient une sécheresse aux conséquences catastrophiques sur récoltes et les pâturages. Il dénonçait également le chômage persistant et la remilitarisation de la zone. Animé par des Touaregs revenus de Libye et de Kidal, le MNLA n'était pas représentatif de l'ensemble de la communauté, qui n'avait toujours pas été consulté quant au bien-fondé de la révolte qui partait, une nouvelle fois, de Kidal.

21 Mouvement laïque, le MNLA réclamait l'autodétermination du peuple de l'Azawad, afin de " prendre en compte les préoccupations de toutes les sensibilités présentes sur le terrain et aussi celles de la population locale ", selon son porte-parole Hama ag Sid'Ahmed. Aussi est-il en rupture avec la composition sociologique des mouvements 
précédents, expliquait-t-il : « la particularité de notre formation réside dans le fait qu'elle se compose d'une jeune élite touarègue et même de certains jeunes arabes lettrés et universitaires. Il y a aussi cette grande force des notables, des chefs des fractions et des tribus qui adhèrent dans leur majorité à cette nouvelle démarche ». Si, au départ, le MNLA était ouvert au dialogue avec le pouvoir central et ses actions étaient pacifiques, il disposait d'une frange militaire composée d'hommes d'expérience, des militaires déserteurs de l'armée régulière et des combattants ayant servi en Libye : ces derniers étaient rentrés au pays lourdement armés et bien entrainés. Emmenés par Ibrahim ag Bahanga, qui n'avait pas cautionné les Accords d'Alger, ils ne devaient intervenir qu'en cas d'échec du dialogue avec le gouvernement malien.

Face à la concentration de troupes dans le nord du Mali décidée par le président Amadou Toumani Touré, qui craignait une nouvelle rébellion, le MNLA déclencha les hostilités. Mieux équipé que les mouvements passés, il s'opposa frontalement à l'armée malienne et non plus sous forme de guérillas. Après de violents combats, il s'empara de Ménaka (17 janvier 2012). Le lendemain, Tessalit tomba. Puis ce fut Aguelhok, où une centaine de soldats maliens faits prisonniers furent égorgés. Les auteurs de ces massacres n'ont pas été identifiés car l'attaque fut menée, d'une part, par les indépendantistes du MNLA qui revendiquèrent la victoire d'Aguelhok, mais nièrent les massacres, et, d'autre part, par les islamistes qui ne les ont pas reconnus, mais sur lesquels pèsent de forts soupçons : ces atrocités qui marquèrent profondément les Maliens s'apparentent en effet davantage aux méthodes d'Al-Qaïda qu'aux agissements habituels des rebelles touaregs. Dès lors, il était évident que les hommes d'AQMI se battaient aux côtés du MNLA. Face à leur poussée, l'armée malienne se replia si bien qu'il n'y eut pas de véritables batailles puisque, quand les rebelles arrivaient dans une localité, les soldats de l'armée régulière l'avaient désertée quelques heures auparavant en abandonnant véhicules blindés, armes et munitions. Kidal, Gao et Tombouctou tombèrent aux mains du MNLA dès la fin du premier trimestre 2012. Cette conquête fut d'autant plus foudroyante qu'un coup d'état s'était produit à Bamako (22 mars 2012) : l'armée malienne, désorganisée et démobilisée, n'opposa aucune résistance à la déferlante touarègue et islamiste. Fort de sa victoire, le MNLA proclama l'indépendance de l'Azawad (6 avril 2012) et le Mali fut, de facto, amputé de plus de la moitié de son territoire.

Le MNLA combattit avec le groupe Ansar ed-Dine, qui apparut au grand jour le 18 mars 2012, soit après les affrontements de Tessalit. S'il s'agit aussi d'indépendantistes, ils divergent du MNLA sur la question de la laïcité voulant appliquer la sharia. Après d'âpres négociations pour se fédérer en un seul mouvement, Iyad ag Ghali, chef d'Ansar ed-Dine et personnage influent des Ifoghas, et Mohammed Najim, chef d'état major du MNLA, ne purent s'entendre quant aux objectifs religieux de leurs mouvements, Iyad ag Ghali refusant de rompre ses liens idéologiques et militaires avec AQMI comme le lui demandait le MNLA. Dès lors, alliés pour affronter l'État malien, les deux mouvements devinrent rivaux : une fois encore, la rébellion touarègue était désunie.

Avec l'aide d'AQMI et du MUJAO, Ansar ed-Dine prit le dessus sur le MNLA, dont les hommes furent chassés des villes. Traversé par de multiples courants, ce dernier vit une partie de ses combattants le déserter pour rejoindre Ansar ed-Dine qui, de plus, offrait des conditions financières plus avantageuses (un nouvel adhérent recevait 300000 francs cfa plus des primes lors d'opérations militaires) en raison de l'implication de son chef dans le convoyage de la drogue et la libération d'otages occidentaux. Opposés à ces deux groupes rebelles, il y avait les 400 à 500 combattants, pour la plupart touaregs, du colonel 
El Hadj ag Gamou, qui commandait la zone de Kidal au début de l'année $2012^{11}$. Restés fidèles au pouvoir central, ces hommes furent stationnés près de Niamey, où ils s'étaient réfugiés après la défaite de l'armée malienne (ils retournèrent au Mali après que les Français eurent repris Gao). Au début de l'année 2013, la communauté touarègue se subdivisait donc en trois blocs : une rébellion «traditionnelle », une rébellion islamiste et des militaires restés fidèles à Bamako. On pourrait ajouter un quatrième bloc, constitué par les populations, premières victimes du conflit. Enfin, les Arabes maliens s'organisaient de leur côté avec la création (mars 2012) d'un nouveau mouvement: le MAA (Mouvement arabe de l'Azawad).

Face à la poussée militaire française, le MNLA, qui entendait redorer un blason bien terni, se déclara prêt à négocier avec les autorités, à aider la France à chasser les islamistes et à récupérer les sept otages. Le 24 janvier 2013, des membres d'Ansar ed-Dine entrèrent en dissidence et créèrent le Mouvement islamique de l'Azawad (MIA), dirigé par Alghabass ag Intalla, fils de l'aménokal des Ifoghas et probable successeur de son père le jour venu, mais aussi négociateur d'Ansar ed-Dine lors des pourparlers de Ouagadougou (fin 2012). Cette personnalité respectée constituait son aile modérée, tandis que Iyad ag Ghaly en était l'aile radicale. Le MIA se dit très vite partisan d'une solution politique à la crise et prêt à combattre les autres groupes «terroristes ». Ce coup de théâtre, qui s'explique en partie par la rapide percée de l'opération Serval, facilita la tâche des armées française, malienne, tchadienne et nigérienne qui progressaient dans le Nord-Est du pays. Désormais, elles n'avaient plus comme ennemi qu'AQMI, le MUJAO et les quelques combattants restés fidèle à Iyad ag Ghali : la majorité de ses hommes rejoignirent en effet le MIA et le MNLA aussi vite qu'ils l'avaient quitté six mois auparavant. L'apparition du MIA, qui prônait des positions radicalement opposées à celles que ses dirigeants défendaient quelques semaines plus tôt, marquait une nouvelle fois la fragmentation de la rébellion et son incapacité à rester unie. Ce volte-face bouleversa le rapport de forces : pragmatique, l'armée française s'appuya sur ces nouveaux alliés touaregs et le solide réseau de renseignements au sol dont elle disposait désormais pour anéantir les « terroristes » et tenter de récupérer les otages, ce qu'elle ne réussit pas à faire.

Face aux succès des militaires français bien épaulés par les troupes tchadiennes et à la restauration progressive de la souveraineté de l'État malien sur des zones dont il avait perdu le contrôle, des chefs de fractions et des notables touaregs créèrent le Haut conseil pour l'unité de l'Azawad (HCUA) à l'issue d'une assemblée générale organisée à Kidal (5 mai 2013). Conscient de la fragilité militaire de la rébellion, son président, Mohammed ag Intalla, déclara " appuyer tous les efforts en vue de trouver par le dialogue une solution politique négociée à la crise que traverse l'Azawad». Celle-ci fut trouvée au terme de négociations menées à Ouagadougou, où un accord de paix a été signé le 18 juin 2013 par le ministre de l'administration territoriale, le colonel Moussa Sinko Coulibaly, par Bilal ag Acheriff, pour le MNLA et Algabass ag Intalla, au nom du HCUA, alors qu'au même moment l'armée malienne s'approchait de Kidal. Cet accord ouvre la voie à l'organisation de l'élection présidentielle sur l'ensemble du territoire national, y compris donc à Kidal, d'où la révolte est partie et où elle se termine une nouvelle fois. De même, tout est parti du clan des Intalla et d'un petit noyau familial déjà au cœur de la rébellion de 1963, et finit sous l'égide de la famille de l'aménokal ${ }^{12}$. 


\section{Les Touaregs nigériens et le conflit malien}

Les "problèmes" touaregs malien et nigérien reposent sur des causes et des revendications proches : ils ne sont donc pas indépendants, même si les rébellions n'ont jamais fusionné. L'histoire montre que ces mouvements se sont d'abord déclenchés au Mali puis ont touché le Niger. Mais, comme en 1963-1964, celui-ci n'a pas été confronté à la révolte, même s'il aurait pu l'être : en mai 2012, Mohamed Anako, ancien responsable de l'Union des forces de la résistance armée (UFRA) de la rébellion des années 1990, déclarait dans un entretien accordé à RFI que « si la CEDEAO intervenait au Mali, il y aura des jeunes Touaregs du Niger, d'Algérie et de Libye qui iront combattre aux côtés de leurs frères du MNLA et ça se généralisera ». L'intervention française a éloigné cette menace, l'armée malienne et les troupes de la CEDEAO ayant été écartées par la France de la région de Kidal jusqu'à ce que la signature de l'accord de paix du 18 juin rende possible leur venue.

Avant de détailler les autres raisons qui expliquent que la rébellion ne se soit pas étendue au Niger bien que ce pays se soit engagé dans le conflit en envoyant 500 soldats dans les régions de Gao et de Ménaka, un bref historique des rébellions touarègues nigériennes paraît utile.

\section{Les rébellions touarègues nigériennes (1991-1995 et 2007-2009)}

C'est dans un contexte politique de démocratisation, pendant les travaux de la Conférence nationale souveraine, que la première rébellion touarègue se déclencha en 1991, soit un an après les évènements de Tchin-Tabaraden. Ceux-ci firent des dizaines de victimes parmi la population touarègue prise sous le feu et les exactions de l'armée nigérienne après l'attaque de la gendarmerie de la localité par un groupe de jeunes touaregs qui protestaient contre l'arrestation arbitraire de certains des leurs et leur sort très précaire (Grégoire, 1999-2010). La situation de la communauté se résumait alors, selon les responsables de la rébellion, «par quelques mots: marginalisation politique, pauvreté absolue et persécution ». Cette marginalisation s'expliquait, comme au Mali, par le manque de représentants de la communauté touarègue au sein de l'appareil d'état et l'insuffisance des infrastructures. Sa principale revendication demandait un statut d'autonomie pour le Nord du Niger. Il n'était pas question d'indépendance, mais d'un système fédéral, qui devait permettre aux régions « revendiquées » de prendre en mains leur développement socio-économique et de bénéficier de la manne financière générée par l'exploitation de l'uranium, dont les mines se trouvent en pays touareg (Arlit). Après une guérilla meurtrière, des premiers accords de paix furent signés à Niamey (24 avril 1995) puis confirmés par un second accord (Alger, 1997) conclu après une reprise des hostilités. Finalement, les mouvements touaregs abandonnèrent leur revendication de «fédéralisme intégral» pour accepter la décentralisation, qui impliquait une réorganisation territoriale et administrative du pays tout en garantissant le maintien de l'unité nationale (Grégoire, 1999 rééd. 2010). Le mouvement avait duré près de quatre ans, soit plus longtemps que celui des Touaregs maliens.

Les difficultés de mise en œuvre de la décentralisation se traduisirent, comme au Mali, par une nouvelle rébellion touarègue, qui éclata avec la création, au début de l'année 2007, du Mouvement des Nigériens pour la justice (MNJ). Il était présidé par Aghali 
Alambo, ancien membre du FLAA (Front de libération de l'Aïr et de l'Azawak) durant la rébellion des années 1990. Contrairement à cette dernière, où les différents fronts faisaient référence à un espace géographique précis et comportaient une forte connotation ethnique, le MNJ se voulait être, au départ, un mouvement national plus large englobant non seulement les régions touarègues, mais aussi le pays toubou, voire d'autres Nigériens qui se sentaient proches de ses aspirations. Le MNJ revendiquait, entre autres, une meilleure application des Accords de paix de 1995, l'instauration d'une véritable démocratie et des mesures économiques pour sortir le pays du marasme économique. Ses effectifs s'étoffèrent puisqu'il fut rejoint par des transfuges de l'armée régulière et des élus locaux. Le MNJ procéda à l'attaque de postes militaires et des combats meurtriers l'opposèrent aux Forces armées nigériennes (FAN) dans le massif de l'Aïr. Il prit aussi pour cible les sites miniers, en prenant en otage des employés d'Areva et un cadre chinois de la compagnie SinoU, filiale de China Nuclear International Uranium Corporation. En représailles à ces opérations de commandos, les FAN s'en prirent aux populations civiles, une nouvelle fois victimes de leurs exactions. Au début de l'année 2009, les affrontements diminuèrent en raison des revers militaires enregistrés par le MNJ et grâce aux pourparlers entamés avec le gouvernement nigérien sous l'égide de la Libye. Bien que le dialogue fût difficile, le président nigérien Tandja Mamadou refusant de négocier avec des hommes qu'il qualifiait de "bandits et de trafiquants de drogue », les fronts touaregs ${ }^{13}$ déposèrent les armes. En contrepartie, le colonel Kadhafi versa d'importantes sommes d'argent à leurs responsables et près de 200000 francs cfa (300 euros) à chaque ex-combattant. Il n'y a eu alors aucun accord politique, contrairement à ce qui s'était passé en 1995, le chef de l'État se contentant d'amnistier les rebelles. Au terme de cette seconde rébellion, le «problème touareg » nigérien n'était toujours pas résolu.

\section{Le Niger à l'écart du conflit malien}

Plusieurs raisons expliquent l'absence d'intrication entre la crise malienne et la rébellion touarègue au Niger, les trois premières concernant les seuls Touaregs, les suivantes le Niger en général :

1. Le président Mahamadou Issoufou a fait de la question touarègue une priorité et s'efforce de pacifier le nord du pays : des forums de la paix ont été organisés par les autorités, en 2012, dans les villes d'Arlit (janvier), de Tchin-Tabaraden (avril) et de Diffa (juillet). Lors de chacun d'entre eux, il fut annoncé des plans de développement pour ces régions.

2. Un système d'intégration a permis à la plupart des dirigeants des rébellions d'occuper des postes administratifs et politiques: ainsi, Brigi Rafini, Touareg d'Iférouane, a été nommé premier ministre ; Rhissa ag Boula, personnage emblématique de la première rébellion, est conseiller du président Issoufou, tandis qu'Aghali Alhambo est conseiller du président de l'assemblée nationale. Mohamed Anako est président du Conseil régional d'Agadez; Rhissa Feltou, ancien cadre du MNJ, est maire d'Agadez; Issoufou ag Maha (MNJ) est maire de Tchirozérine. Ces anciens rebelles ont donc d'importantes fonctions, l'idée étant de s'appuyer sur eux pour pacifier le Nord. Rien ne dit cependant qu'ils aient une réelle emprise sur les nombreux jeunes revenus de Libye, dont la réinsertion est rendue malaisée par l'absence de perspective économique et d'emplois.

3. Malgré ses imperfections, la mise en place de la décentralisation, qui est une concession faite à la rébellion, a permis d'associer les populations à la gestion de leur région et des villes. Cette réforme a donné lieu à de nouveaux découpages administratifs, le Niger étant subdivisé en 8 régions, 36 départements et 265 communes (2004). Les régions et les 
communes disposent désormais de leur propre budget géré par des Conseils communaux et régionaux qui élisent un conseil exécutif. Des Touaregs ont ainsi accédé à des postes de responsabilités et pris des décisions en matière de développement local : le code minier de 2007 attribue en effet $15 \%$ des recettes minières aux régions concernées par l'exploitation de matières premières, ce qui profite aux zones touarègues où se trouvent les sites uranifères.

4. Le fait qu'AQMI ne soit pas réfugié au Niger joue également : l'organisation a été un puissant élément de déstabilisation au Mali. Toutefois, AQMI ne se s'est pas privé de faire des incursions au Niger pour se ravitailler en otages comme il le fit, en septembre 2010, sur le site minier d'Areva à Arlit puis, en janvier 2011, à Niamey (enlèvement de deux jeunes gens décédés lorsque l'armée française tenta de les libérer à la frontière malienne). Le Niger constituait alors pour AQMI une zone d'approvisionnement en otages et non un lieu de détention.

5. Le Niger a été aussi moins concerné que son voisin par le trafic de drogue : celui-ci n'y a pas pris la même ampleur, même si son territoire est traversé par des pistes d'acheminement de la cocaïne qui partent de l'extrême nord-est du Mali pour finir en Libye (des Touaregs nigériens participent à son convoyage). De ce fait, l'appareil d'État a été moins touché par la corruption que l'État malien, où l'activité s'est développée avec la complicité de hauts personnages proches du pouvoir, de l'armée et de la douane. Cependant, le conflit malien pourrait inciter les trafiquants latino-américains à explorer de nouvelles routes qui traverseraient le Nigeria puis l'Est du Niger pour regagner la Libye et l'Égypte.

6. L'État nigérien est plus fort que l'État malien, très affaibli sous la présidence d'Amani Toumani Touré : le président Mamadou Tanja, jusqu'à ce qu'il se livre à un coup d'état constitutionnel pour se maintenir au pouvoir (2009), tenait bien en mains les rênes du pays. Son successeur Mahamadou Issoufou, démocratiquement élu le 11 mars 2011, semble avoir la confiance de la population et le soutien d'une armée forte de près de 30000 hommes. Sur le plan diplomatique, il a fait preuve d'une grande fermeté se déclarant, dès le début du conflit, partisan d'une intervention étrangère (CEDEAO) au Mali.

7. La situation économique du Niger a tendance à s'améliorer en raison du surcroît de recettes tirées des exportations d'uranium, dont le prix payé par Areva a été multiplié par 3,5 entre 2004 et 2011, passant de 21000 francs cfa / kg à 71 000. Parallèlement, la production d'Areva augmente et est complétée par la mise en route d'une nouvelle mine exploitée par une société chinoise. D'ici quatre ans, elle devrait fortement croître avec l'exploitation du site d'Imouraren, concédé à Areva (Grégoire, 2011). Les moyens de l'État devraient donc être plus importants, ce qui lui permettra d'investir à l'intérieur du pays ${ }^{14}$.

8. Enfin et sans doute surtout, la France a de gros intérêts économiques au Niger, ce qui n'est pas le cas au Mali. Les mines d'Arlit assurent $30 \%$ de son approvisionnement en uranium, chiffre qui passera à $50 \%$ quand la mine d'Imouraren dont l'ouverture est prévue en 2015 atteindra son plein régime (2017). Les sites d'Arlit et d'Imouraren sont donc stratégiques pour Paris qui a envoyé, peu après la prise d'otages d'In Amenas (Algérie), des forces spéciales pour les sécuriser aux côtés de l'armée nigérienne. Dans ce contexte, l'exploitation de l'uranium par la France mais aussi désormais par la Chine constitue pour le Niger une «force de dissuasion", les islamistes sachant qu'une perturbation des exportations entraînerait une riposte militaire immédiate de la France.

32 Ces raisons expliquent que le Niger ait été relativement épargné par le conflit malien. Les attaques perpétrées (23 mai 2013) par les "Signataires du sang» de Mokhtar Ben Belmokhtar et le MUJAO contre l'usine de la SOMAÏR d'Arlit et la caserne de l'armée nigérienne d'Agadez rappellent l'opération menée contre le site gazier algérien d'In Amenas (16-19 janvier 2013). Dans les deux cas, il s'agit d'opérations de commandos destinées à punir des États qui soutiennent la France et résistent aux islamistes : chassés 
du Mali, ils ne se sont pas réfugiés en Algérie ni au Niger, mais dans la vallée d'Ubari, c'est-à-dire dans le Sud libyen vaste région qui échappe au contrôle de Tripoli et devient leur nouveau sanctuaire.

Pour le Niger, le principal élément d'inquiétude tient à la présence sur son sol de nationaux qui ont rejoint AQMI et le MUJAO, au contact desquels ils ont appris le maniement des armes. Certes, les thèses islamistes ne rencontrent guère l'adhésion des populations, qu'elles soient du nord ou du sud du pays, et leur influence reste minime, mais la constitution de cellules islamistes nigériennes est préoccupante : certains groupes arabes de la région de Tasara ont ainsi noué des liens économiques (trafic de drogue) et religieux avec le MUJAO. Celui-ci est en relation avec la secte Boko Haram (appellation que l'on peut traduire par « interdire les livres » c'est-à-dire l'éducation occidentale) qui sévit au Nigéria. Implantée au départ à Maïduguri, la secte étend son influence dans tout le Nord de la fédération et même à la région de Diffa, en raison de sa proximité et de la perméabilité de la frontière. Celle-ci a servi de zone de repli lorsque ses partisans furent violemment combattus par l'armée nigériane (2009). Depuis lors, Boko Haram a radicalisé son idéologie et son action (prises d'otages) ${ }^{15}$. Pour les autorités nigériennes, la secte constitue une source d'inquiétude aussi importante qu'AQMI : un petit groupe a ainsi fait évader ( $1^{\text {er }}$ juin) de la prison de Niamey Chedani, condamné pour l'assassinat de quatre Saoudiens et d'un Américain, et plusieurs autres de ses adeptes.

Le Niger se trouve donc dans une situation délicate étant pris en tenailles entre, au Nord les islamistes, dont Mokhtar Ben Belmokhtar, partis se réfugier dans la région de Sabha (Libye), à l'Ouest les quelques partisans d'AQMI et du MUJAO encore opérationnels au Mali et, au Sud Boko Haram actuellement combattu par le gouvernement nigérian.

\section{Conclusion}

La crise malienne apparaît comme un dégât collatéral d'événements qui se sont déroulés d'abord en Algérie puis en Libye, où le colonel Kadhafi pourchassait les islamistes. À travers l'opération Serval, la France a tenté de réparer les dégâts faits au Sud du Sahara par son intervention militaire en Libye ${ }^{16}$. Si elle fut un succès militaire, Paris va devoir maintenir durablement une présence militaire au Nord du Mali afin d'empêcher un éventuel retour des «terroristes ». Le gouvernement français, avec la Minusma (Mission des Nations Unies pour le Mali), devra aussi s'impliquer dans la question très délicate du retour de l'armée malienne à Kidal et du cantonnement des combattants touaregs.

Ces pages ont montré l'incapacité des mouvements islamistes et touaregs à présenter un front uni en raison de leurs divergences: les premiers sont traversés par des rivalités politiques, économiques et personnelles tandis que les seconds ont été affectés, comme lors des précédentes rébellions, par la versatilité des alliances et la forte mobilité des combattants qui passent sans cesse d'un front à un autre. On retrouve là un trait inhérent aux rébellions touarègues. Aussi ce conflit a-t-il été fait d'éphémères alliances, de concurrences, de divisions et de ruptures qui ont finalement conduit ou accéléré son échec. Sans doute manque-t-il à la communauté touarègue un véritable leader capable de la mobiliser autour de lui et d'un projet commun: les rébellions ont fait émerger des générations de chefs de guerre tant au Mali qu'au Niger, mais pas de véritable chef politique comme ce peut être le cas dans d'autres régions, le système politique traditionnel marqué par la segmentarité freinant une telle dynamique. 

ampleur plus grande que les précédentes en raison de la présence des islamistes et d'une conjoncture régionale particulièrement instable. Une nouvelle fois, l'avenir se pose en termes de développement afin que la communauté touarègue trouve enfin sa place au sein de la nation malienne et s'insère dans l'économie moderne. Mais comment faire pour l'aider à y parvenir?

\section{BIBLIOGRAPHIE}

Bisson J., 2003. Mythes et réalités d'un désert convoité : le Sahara. Paris, L'Harmattan, 480 p.

Boilley P., 1999 rééd. 2012. Les Touaregs Kel Adagh : dépendances et révoltes, du Soudan français au Mali contemporain. Paris, Karthala, 644 p.

Bourgeot A., 2011. Sahara de tous les enjeux. Hérodote, Géopolitique du Sahara, n 142, Paris, La Découverte, p. 42-77.

Bourgeot A., 1996. Les rébellions touarègues : une cause perdue? Afrique contemporaine, $\mathrm{n}^{\circ} 180$, Paris, p. 99-115.

Champin Ch., 2010. Afrique noire poudre blanche. L'Afrique sous le coude des cartels de la drogue. Bruxelles, André Versaille éditeur, $160 \mathrm{p}$.

Durand G., 2011. L'organisation d'Al-Qaïda au Maghreb islamique. Réalité ou manipulations? Paris, L'Harmattan, $122 \mathrm{p}$.

Grégoire E., Bourgeot A., 2011. Désordre, pouvoirs et recompositions territoriales au Sahara. Hérodote, Géopolitique du Sahara, n 142, Paris, La Découverte, p. 3-11.

Grégoire E., 1999 rééd. 2010. Touaregs du Niger, Le destin d'un mythe. Paris, Karthala, 359 p.

Grégoire E., 2011. Niger : un État à forte teneur en uranium. Hérodote, Géopolitique du Sahara, n ○ 142, Paris, La Découverte, p. 206-225.

Hureiki J., 2003. Essai sur les origines des Touaregs. Paris, Karthala, 764 p.

Lecoq B., 2002. That desert is our country. Tuareg rebellions and competing nationalism in contemporary Mali (1946-1996). Amsterdam, Amsterdam-School for Social Science Research, 338 p.

Loncle F., Plagnol H., 2012. Le Sahel pris en otage. Rapport d'information n 4431, Assemblée nationale, Commission des affaires étrangères, Paris, $121 \mathrm{p}$.

Scheele J., 2012. Smugglers and Saints of the Sahara, Regional Connectivity in the Twentieth Century. Cambridge University Press, 270 p.

Simon J., 2011. Le Sahel comme espace de transit des stupéfiants. Acteurs et conséquences politiques. Hérodote, Géopolitique du Sahara, n 142, Paris, La Découverte, p. 125-142.

EchoGéo, Sur le Vif 


\section{NOTES}

1. Ils craignaient que ne soit remis en cause le statu quo établi avec les militaires algériens qui ne les inquiétaient pas tant que leurs opérations restaient localisées hors du territoire national.

2. Du rififi chez les émirs d'AQMI, in Maghreb confidentiel, $\mathrm{n}^{\circ}$ 1001, 12 janvier 2012.

3. Selon le World Drug Report (2010) de l'office des Nations unies contre la drogue et le crime (ONUDC), $15 \%$ de la production mondiale de cocaïne transiterait par l'Afrique de l'Ouest soit entre 80 et $100 \mathrm{t} / \mathrm{an}$ (Simon, 2011).

4. Les émirs recoururent à d'autres sous-traitants pour enlever deux Français à Hombori (24 novembre 2011) ; ils leur auraient versé 10 millions de francs cfa $(15000 €)$ cf. La Lettre du Continent, $\mathrm{n}^{\circ} 627,19$ janvier 2012.

5. Celui-ci a été tué en mars 2013 par les troupes françaises ou tchadiennes dans le massif des Ifoghas. Les otages qu'il détenait seraient aux mains de Yahia Djouadi en Algérie.

6. Bien qu'occulté, l'appât du gain motive les émirs : Abou Zeid possédait des biens en Algérie et une grosse somme d'argent en liquide acquise à partir des rançons et du trafic de drogue (cf. Sahara Médias, 12 mars 2013).

7. Cette lettre attribuée à Abdel Malek Droukdal est très certainement un faux document écrit par des partisans du MNLA. Elle mérite cependant d'être évoquée car elle propose une intéressante analyse des évènements.

8. L'amenokal est le chef d'un ensemble de tiwsaten (sing. tawset), groupes politiques et sociaux fondés sur la parenté lignagère et réunis au sein d'un ettebel (d'une confédération).

9. Ce terme vient de chômeur et désigne les nombreux jeunes sans travail partis en exil en Libye.

10. L'Algérie ne s'imposait plus comme seul médiateur en raison de l'influence grandissante, comme au Niger, de la Libye.

11. El Hadj ag Gamou est un imrad des Ifoghas, ancien leader de la rébellion de 1990, devenu ennemi juré de Iyad Ag Ghali.

12. Les membres du MNLA seront cantonnés, mais ne déposeront pas immédiatement les armes ; ils bénéficieront d'un processus dit DDR (désarmement, démobilisation, réinsertion socioéconomique).

13. Comme lors de la précédente rébellion marquée par la scission de plusieurs fronts, le MNJ donna naissance à des groupes dissidents: le 30 juin 2008 fut créé le Front des forces de Redressement (FFR) avec à sa tête Rhissa ag Boula. En mars 2009, Sidi Aklou fonda le Front patriotique nigérien (FPN).

14. Le budget du Niger a presque doublé entre 2005 (500 milliards de francs cfa) et 2011 (940 milliards).

15. Boko Haram se fragmenta et donna naissance à Ansaru al-Muslimin Fi Bilad al-Sudan (les défenseurs des musulmans en Afrique noire).

16. L’Afrique du Sud, la Russie et la Chine la critiquèrent jugeant qu'elle aurait été gérée de façon irresponsable, sans se soucier des répercussions néfastes pour la région (Loncle \& Plagnol, 2012). 


\section{RÉSUMÉS}

Au moment où le conflit malien semble trouver une solution (juin 2013), cet article revient sur les alliances, de rivalités et de ruptures entre et au sein des différents mouvements à l'origine de la crise. Inhérentes à leurs dynamiques internes, ces divisions expliquent en partie leur récent échec politique, militaire et religieux.

L'auteur retrace l'implantation des islamistes dans le Nord Mali et analyse leurs dissensions. Il examine ensuite les revendications et les divisions des mouvements touaregs, qui se sont radicalisés depuis les précédentes rébellions allant pour certains (MNLA) jusqu'à proclamer l'indépendance de l'Azawad et pour d'autres (Anser ed-Dine) à vouloir créer par la lutte armée un État islamique touareg appliquant la sharia. Enfin, il se demande pourquoi les Touaregs nigériens n'ont pas pris les armes alors que les rébellions touarègues malienne et nigérienne présentent de grandes similitudes.

At the time when the conflict Malian seems to find a solution (June 2013), this article reconsiders alliances, competitions and ruptures between and within the various movements at the origin of the crisis. Inherent in their internal dynamics, the divisions partly explain why they recently failed on the political, military and religious levels.

The author recalls the establishment of the Islamists in North Mali and examines their dissensions. He then analyses the claims and the divisions in the Tuareg movements, which radicalized since the preceding rebellions, as some decided Azawad be independent and while others (Anser ed-Dine) decided to set up an Islamic Tuareg state through armed conflict and to apply sharia. Lastly, he wonders why the Tuareg from Niger did not take up arms while the Malian and Nigeria Tuareg rebels show great similarities

\section{INDEX}

Mots-clés : rébellions touarègues maliennes, mouvements islamistes, opération Serval, versatilité des alliances, Niger

Keywords : Malian Tuareg rebellions, Islamist movements, Serval operation, fickleness of alliances, Niger

\section{AUTEUR}

\section{EMMANUEL GRÉGOIRE}

Emmanuel Grégoire, emmanuel.gregoire@ird.fr, est Directeur de recherche IRD, Centre d'études africaines (CEAf). 